\title{
Optical Recordings of the Cortical Response to Whisker Stimulation Before and After the Addition of an Epileptogenic Agent
}

\author{
Jill A. London, ${ }^{a}$ Lawrence B. Cohen, and Jian-Young Wu \\ Department of Cellular and Molecular Physiology, Yale University School of Medicine, New Haven, Connecticut 06510
}

\begin{abstract}
Optical recording methods using voltage-sensitive dyes were used to monitor activity in rat somatosensory cortex. We measured evoked signals in response to whisker stimulation before (control) and after the addition of the epileptogenic agent, bicuculline, and also detected spontaneous interictal events that occurred after bicuculline. Bicuculline led to an increase in the size, duration, cortical extent, and, surprisingly, the latency of the evoked responses. These enhanced evoked responses appeared to originate in the region of the control response and propagate outward. In contrast, the spontaneous signals appeared to originate at random cortical positions and had a more variable cortical extent. A transition signal measured just after the addition of bicuculline was larger than the control response but localized and rapid in time course. In most cases, there was a good correlation between the optical recordings and field potential measurements made with a ball electrode on the cortical surface, but there were occasional instances where the optical signal disappeared while the ball electrode signal was unchanged.
\end{abstract}

Optical methods for monitoring neuron activity might be useful for determining the origin and the spatial spread of epileptiform events because these methods allow simultaneous monitoring of cortical activity at many sites. Early attempts to use multisite imaging to measure epileptic activity in cortex (Schuette et al., 1974) involved mcasurcments of NADH fluorescence, an indicator of the intracellular metabolic state. Optical measurements during epileptic events from single sites in cortex have been reported by several laboratories (Jobsis et al., 1971; Rosenthal and Jobsis, 1971; O'Connor et al., 1972; Lewis et al., 1974; Evans and Smith, 1987). While NADH fluorescence was measured in most of these experiments, Evans and Smith (1987) also measured the fluorescence of a polential-sensitive oxonol dye. However, they suggested that this signal was reporting changes in mitochondrial membrane potential and thus both the NADH and the oxonol signals were interpreted in terms of cortical energy metabolism.

More recently, Orbach et al. (1985) showed that the fluorescence from cortex stained with a potential-sensitive styryl dye

Received Aug. 22, 1988; revised Nov. 9, 1988; accepted Nov. 21, 1988.

We thank Dr. A. Grinvald and R. Hildesheim for informing us that RH795 might prove useful and for providing the styryl dyes. Supported by NIH Grant NS08437.

a Present address: Department of BioStructure and Function, University of Connecticut Hcalth Ccnter, Farmington, CT.

Copyright (C) 1989 Society for Neuroscience $0270-6474 / 89 / 062182-09 \$ 02.00 / 0$ could be used to form a rough image of cortical activity in response to whisker movements. It seems likely that the styryl signals represented the average change in membrane potential of the membranes imaged onto each photodetector. Orbach et al. (1985) also found that applying the epileptogenic agent bicuculline to the cortex led to larger and longer-lasting signals which occurred over an extended area of cortex. Bicuculline is a GABA antagonist that increases the size of evoked responses as recorded with extracellular electrodes (Zemon et al., 1980) and induces spontaneous interictal epileptiform discharges. We have pursued these findings in an attempt to determine the relationship of the optical signals that occur in normal cortex (control responses) and in cortex after the addition of bicuculline. We also hoped to clarify the relationship between epileptic events evoked by whisker stimulation and those that occurred spontaneously in the presence of bicuculline. The measurements we describe were made with a recording time resolution of about $1 \mathrm{msec}$, more than 100 times faster than those used for measuring cortical energy metabolism.

Preliminary reports of our experiments have appeared (London et al., 1986, 1987). For reviews of multisite optical recordings using voltage-sensitive dyes see Cohen and Salzberg (1978), Bashford (1981), Salzberg(1983), Dasheiff(1988), Orbach (1988), and Grinvald et al. (1988).

\section{Materials and Methods}

Optical methods for measuring changes in fluorescence from the stained cortex were modifications of those previously published (Grinvald et al., 1981; Orbach and Cohen, 1983; Orbach et al., 1985; Cohen and Lesher, 1986). Epi-illumination was used, and fluorescence was measured using 124 elements of a $12 \times 12$ photodiode array (Fig. 1). A nominal $2.5 \times, 0.08 \mathrm{NA}$ microscope objective formed an image of the cortex on the diode array. The actual magnification of the objective was $3.3 \times$. With this magnification, the centers of neighboring diodes will receive light from points on the cortex that are separated by $450 \mu \mathrm{m}$; the total area of cortex that was monitored was $5.5 \times 5.5 \mathrm{~mm}$, about 5 times larger than was cxamincd previously (Orbach et al., 1985). The output of each detector was amplified, digitized, and stored in a DEC PDP 11/34 computer. For analysis the data were transferred to a Motorola VME 1000 computer. The programs, in 68020 assembler and PASCAL, running under VERSAdos, are available from the authors. An $\mathrm{Ag} / \mathrm{AgCl}_{2}$ ball electrode was positioned on the dura just outside the field of view of the photodiode array (at the upper right corner in Figs. 2 and 7).

Noise reduction. The raw fluorescence measurements contain large artifacts due to the movement of the cortex as a consequence of the pulsatile blood flow and respiratory activity. In addition to subtraction methods to reduce heartbeat artifacts (Orbach et al., 1985), we also eliminated the noise due to respiratory movements. The rats were paralyzed and artificially respirated; during each trial the computer turned off the respirator for about $1.5 \mathrm{sec}$. Even after these procedures, signal averaging was needed to detect the control signals; averaging was not 
used for measurements of signals after the addition of bicuculline.

The low- and high-frequency response of the system was limited electronically to $1.5-300 \mathrm{~Hz}$. Additional high-frequency filtering was carried out digitally using a 1-2-1 digital smoothing routine. Ten passes of this routine reduced the high-frequency cut-off to $20 \mathrm{~Hz}$. The effects of this filtering are illustrated in figure $1 C$ of Orbach et al. (1985). The most prominent distortion will be a reduction in the duration of slow signals, i.e., those measured after the addition of bicuculline.

Preparation. Male, albino Wistar SPF rats, $180-300 \mathrm{gm}$, were anesthetizcd with Equithesine $(4 \mathrm{ml} / \mathrm{kg})$. A $6 \times 6 \mathrm{~mm}$ craniotomy was performed over the somatosensory cortex. The dorsal edge of the craniotomy was $3 \mathrm{~mm}$ from the midline, the caudal edge was at lambda. The dura was left intact. Previous experiments (Orbach et al., 1985) suggested that in the rat, where the dura is transparent, the presence of the dura did not seem to affect signal size or distribution. A well to hold the staining solution was made with Kadon dental cement applied to the surrounding bone. The cortex was stained for $1 \mathrm{hr}$ using a $1 \mathrm{mg} / \mathrm{ml}$ solution of the styryl dye RH795 (Grinvald et al., 1986) in saline. The dye was continuously and gently swirled over the dura using a perfusion pump. We used this dye in place of the previously used RH414 because RH4 14 at concentrations higher than $0.1 \mathrm{mg} / \mathrm{ml}$ caused an irreversible constriction of arteries on the surface of the cortex. We thank Amiram Grinvald for telling us about this problem and suggesting the use of RH795. After staining, the rat was respirated using a IIarvard small animal ventilator $(110 / \mathrm{min}$ with a volume of $3 \mathrm{ml})$ and then paralyzed using $100 \mathrm{mg} / \mathrm{kg}$ Flaxedil. A $1 \mathrm{~mm}$ bicuculline solution was prepared as previously described (Zemon et al., 1980). The bicuculline was applied by placing a drop of the solution over the entire area of the cortex exposed by the craniotomy. With the objective we used, most of this exposed area was imaged onto the array. A heating pad was used to regulate the rectal temperature of the animal between 36 and $39^{\circ} \mathrm{C}$.

Experimental protocol. The following procedure was used for each evoked trial. The incident light shutter was opened. One second later, the respirator was turned off. After another $0.5 \mathrm{sec}$ the computer triggered the data acquisition on the next upswing of the largest electrocardiogram (ECG) signal. No stimulation was given during this recording. Following this first recording the computer again looked for the ECG signal and initiated a sccond recording. During the second recording the whisker was moved. At the end of the second recording, the respirator was turned on and the shutter was closed. The first recording was then subtracted from the second; the subtracted result is called a single trial. The whisker movement (Orbach et al., 1985) was kept constant for all evoked responses on a given preparation. There was no whisker movement during recordings of spontaneous events. The interstimulus interval for the signal-averaged control trials was usually $7 \mathrm{sec}$. Since it took approximately $2 \mathrm{~min}$ to display and store a set of results, recordings after bicuculline addition were made about once every 2-3 min.

\section{Results}

Figure 2 illustrates the evoked optical signals recorded from somatosensory cortex in response to a whisker movement before (top) and after (bottom) the addition of $1 \mathrm{~mm}$ bicuculline. The time of the beginning of the whisker movement is indicated by the small arrows under 3 of the detectors in both panels and under the ball electrode recordings shown in the upper-left corner of both panels. Bicuculline affects the response to whisker movement in 3 ways. First, the control signal is present in a small area of cortex, while after bicuculline the signal is found on almost every photodetector. Second, the signal after bicuculline is larger. In Figure 2, the fractional change $(\Delta F / F)$ of the control signal was about $5 \times 10^{-4}$, while after bicuculline the signal was 6 times larger. On average ( 8 preparations), the addition of bicuculline led to an increase in signal size by a factor of $13 \pm 2$ (mean $\pm \mathrm{SEM}$ ). Because of the much larger signal size, the optical recordings made after the addition of bicuculline did not requirc signal avcraging. All of these results come from single trials. Third, the control signal is briefer; note that the time base for the top panel is only half as long as the bottom panel. Similar differences in response characteristics were ob-

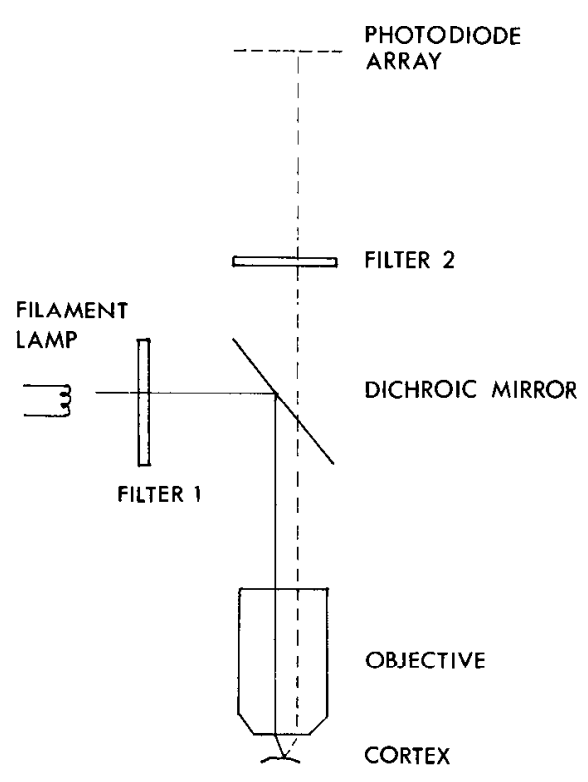

Figure 1. Schematic diagram of the optical apparatus for epifluorescence. Light from a tungsten halogen lamp was passed through a heat filter (not shown) and a $520 \pm 45 \mathrm{~nm}$ interference filter before being reflected down onto the cortex by a dichroic mirror with a half-reflectance wavelength of $570 \mathrm{~nm}$. A Schott RG610 long-wavelength pass filter was used to block the scattered and reflected light while passing the longer-wavelength fluorescent light to 124 elements of a $12 \times 12$ photodiode array. Each element of the array was $1.4 \times 1.4 \mathrm{~mm}$ with a center-to-center spacing of $1.5 \mathrm{~mm}$. The photodiode array was placed in the plane where the objective makes its image.

tained in every preparation in which these 3 comparisons could be made.

Figure 3 compares the time course of the evoked signals before and after bicuculline in 2 preparations using an expanded time scalc. The control signal is briefer and occurs with a shorter latency after the beginning of the whisker movement. In 8 preparations we measured the time between the beginning of whisker movement and the time for the optical signal to reach its halfheight. For the control signals the average latency was $32 \pm 5$ msec; after bicuculline the latency increased to $69 \pm 12 \mathrm{msec}$.

While the evoked signal illustrated in the bottom panel of Figure 2 appears to occur at the same time on all the detectors, when an expanded time base is used, the signal is found first in that area of cortex where the control signals were found (Fig. 4). Figure 4 illustrates the outputs of 4 groups of 9 detectors. The signal from region 1 (the detector labeled 1 and the 8 surrounding detectors) occurs earlier than the signals from 3 other regions of the array. Region 1 is where the control signal is found (Fig. 2, top panel). We determined the location of the shortest latency evoked epileptic signal in 5 preparations in which the location of the control signal was also determined. In 3 preparations, including the one illustrated in Figures 2 and 4, the evoked epileptic signal appeared to originate in the same area of cortex where the control signal was found. In the fourth preparation, the evoked response appeared simultaneously in an area of cortex that was somewhat larger than the control response area. In the fifth preparation, the evoked response after bicuculline appeared simultaneously (within the accuracy of our measurements, ca. $1.5 \mathrm{msec})$ on the entire cortical area $(5.5 \times$ $5.5 \mathrm{~mm}$ ) that was monitored.

In the experiment illustrated in Figure 4, the apparent prop- 


\section{BEFORE BICUCULLINE}

Figure 2. Optical recordings from rat somatosensory cortex in response to whisker stimulation before (top) and 7 min after (bottom) the addition of $1 \mathrm{~mm}$ bicuculline to the surface of the cortex. Each trace represents the output of one photodiode. The control signals measured before the addition of bicuculline were much smaller and occurred over a more restricted area. Since both signals are elicited by whisker movements they are referred to as evoked signals. The pixels are arranged so that their relative position in the figure corresponds to the relative position of the areas of cortex imaged onto the array. In this figure (as well as Figs. 5 and 7), top is medial, bottom is lateral, left is caudal, and right is rostral. The time of the beginning of the whisker movement is indicated by the arrowheads under 3 of the detectors in both panels and under the ball electrode recordings shown in the upper-left corner of both panels. The outputs of only 108 pixels of the photodiode array are shown; the pixels on the bottom row did not have signals and were omitted. In the upper-left corner is shown the simultaneously measured ball electrode recording. In the recordings presented in Figures 2 and 5 , as many as 4 of the photodiode amplifiers were not functioning. For those pixels, the data displayed is the mean of the abutting 4 pixels. In this and subsequent figures, the length of the vertical line to the right of the traces represents the stated value of the change in fluorescence divided by the resting fluorescence $(\Delta F / F)$. In this figure, the value holds for the detectors in the center of the field. For the detectors at the edge, the bar would be smaller because less light reaches those detectors. In addition to the high-frequency filtering provided by the amplifier system, the traces were smoothed with 10 passes of a 1-2-1 digital smoothing routine. Top, The duration of the traces is $135 \mathrm{msec} ; 32$ trials were averaged. Bottom, The duration of the traces is $270 \mathrm{msec}$; this result is from a single trial.

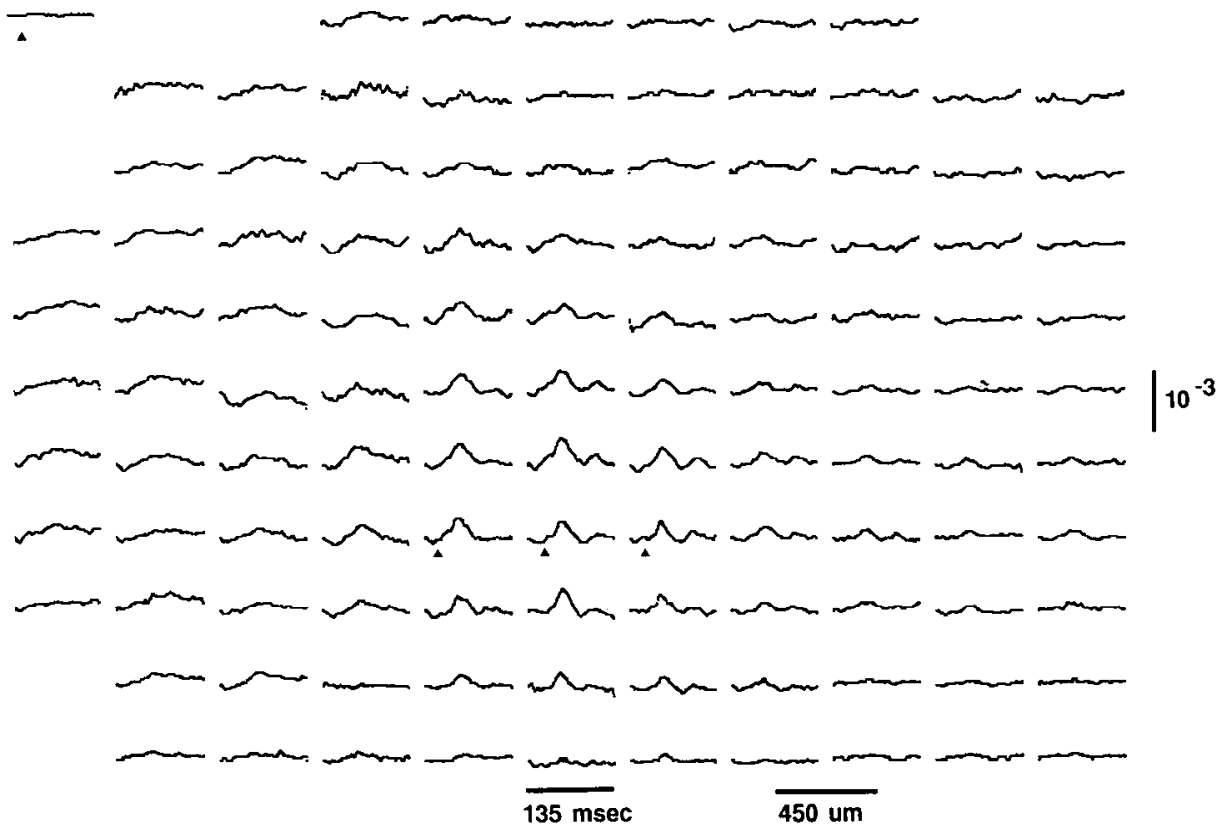

AFTER BICUCULLINE

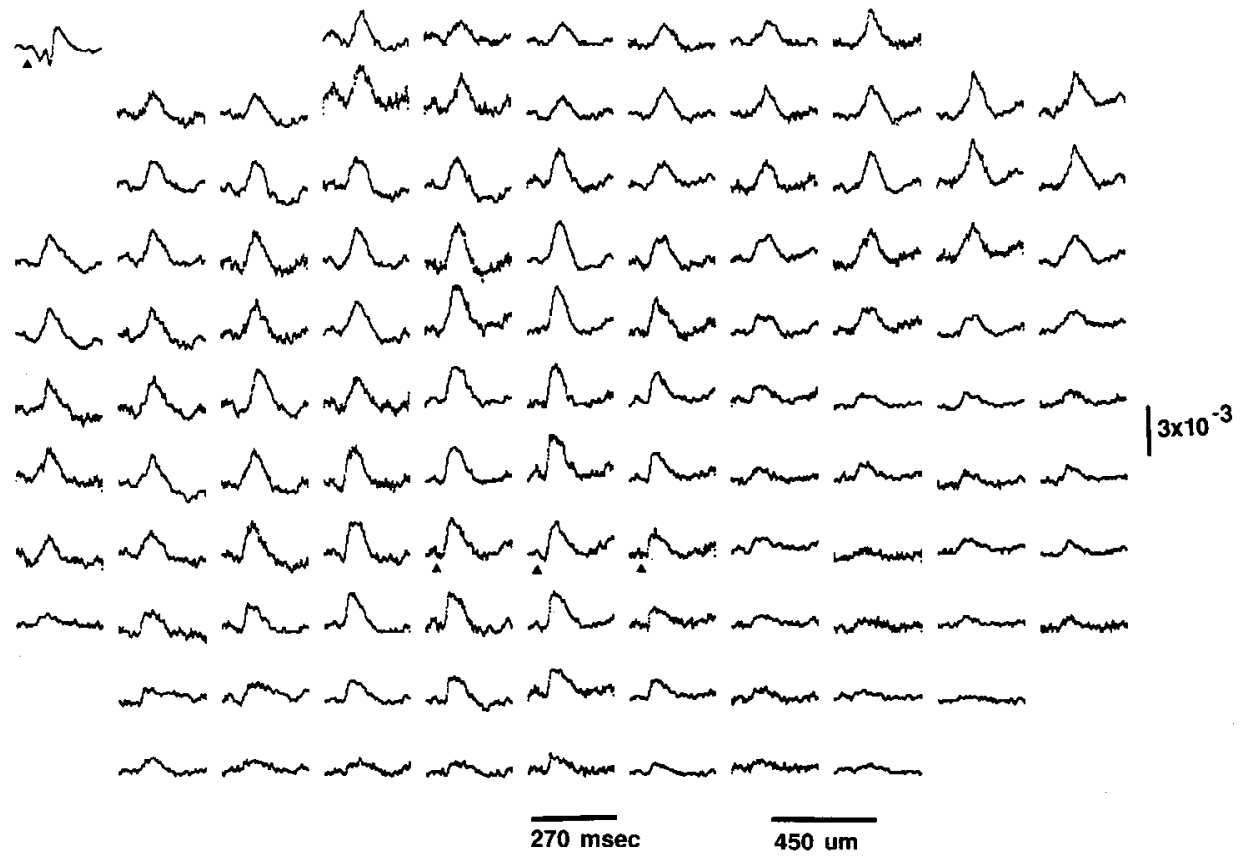

agation velocity of the signal does not appear to be directly proportional to the cortical distance from the origin (location 1). However, in other preparations, propagation velocity was proportional to distance. The apparent propagation velocities of the half-height of the upstroke of the signals in Figure 4 were calculated on the assumption that the signal originates in region 1 and propagates from there directly to positions 2,3 , and 4 . Values from 0.1 to $0.3 \mathrm{~m} / \mathrm{sec}$ were obtained. The average velocity in the 3 directions was $0.2 \mathrm{~m} / \mathrm{sec}$. The mean of the averages from 8 preparations was $0.6 \pm 0.1 \mathrm{~m} / \mathrm{sec}$. This mean does not include the preparation mentioned above in which the evoked signal appeared simultaneously over the monitored area.
In this unusual case, the propagation velocity was at least 3 $\mathrm{m} / \mathrm{sec}$.

The response to whisker movement that occurred after the addition of bicuculline could be measured several times on the same preparation. Figure 5 illustrates partial results of 8 such measurements. The third trial after the addition of bicuculline is shown nearly completely; for the other 7 trials, the outputs of only the fourth row of detectors is shown. The small arrows under 8 detectors in trial 3 mark the detectors that recorded control signals with amplitudes greater than $50 \%$ of the largest control signal. The response to whisker movement appeared to be similar in size and time course in trials $2-8$. In addition, in 
EVOKED RESPONSES

EXPERIMENT \# 7

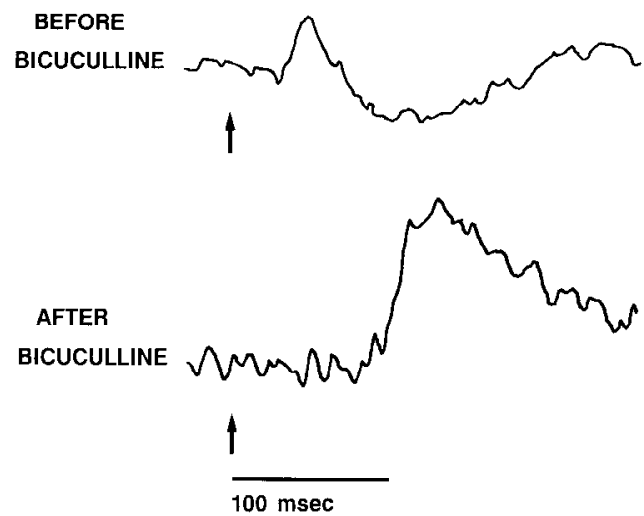

Figure 3. Comparison of the time course of the control evoked response and the evoked response after the addition of bicuculline. The control responses in both experiments are smaller, briefer, and occur with a shorter latency. Each trace is the average of 9 adjacent detectors centered in the area of the control response. For clarity of presentation, the size of the control signals have been increased relative to the recordings made after bicuculline. When expressed in terms of signal size per trial, the signals after bicuculline were 16 and 12 times larger than the control signals in experiments 7 and 16 , respectively. The signal-to-noise ratios obtained after bicuculline are not large enough to rule out the continued presence of the control signal in those recordings. For the control signals, 128 trials were averaged in experiment 7 and 32 trials were averaged in experiment 16 . No averaging was used for the trials after bicuculline. these 7 cases the response appeared first in the region indicated by the small arrows, and only later elsewhere on the cortex. In these 7 trials, the latency to half-height of the signals at the apparent origin was constant, $18.7 \pm 0.7 \mathrm{msec}$. In addition the half-height of the response at the apparent origin preceded the response from regions 1,4 , and 3 (Fig. 4) by $4.8 \pm 0.4,5.9 \pm$ 0.4 , and $9.6 \pm 0.9 \mathrm{msec}$, respectively. Thus, in this preparation, the propagation velocity was also constant from trial to trial. In a second preparation there was more variability between trials.

However, the first response (Fig. 5, trial 1), measured $2 \mathrm{~min}$ after the addition of bicuculline, was quite different from those recorded in trials $2-8$. This first post-bicuculline response had a rapid time course, and it appeared only on the detectors on the right-hand side of the array, in fact, on just those detectors that had control signals. Data from this experiment are shown on an expanded time scale in Figure 6. The time course of the signal in a control trial and the first and third trials after bicuculline are compared. The traces are drawn to show the relative signal size in a single trial for all 3 recordings. The control signal is relatively small. The signal obtained in the first trial after bicuculline (transition signal) has a time course quite similar to the control signal. Thus, the bicuculline first increased the signal size (by a factor of about 7) without affecting its time course or distribution over the cortex. It is only in the next (third) trial, 6 min later, that the signal has the characteristic slower time course, longer latency, and propagation over the whole area of cortex.

In addition to the signals evoked by whisker stimulation, we also detected optical signals from spontaneous epileptiform events after the addition of bicuculline. These interictal signals did not share the regularity we found for the evoked response. Two such spontaneous events, from the same preparation used for Figure 5 are shown in Figure 7 . The bottom panel illustrates a spontaneous event that was largest in the upper-right portion of the array. In the upper panel there was a spontaneous event whose spatial extent was very similar to the evoked response shown in Figure 5. However, in this case, the signal did not originate in the upper right as did the evoked responses but occurred first on the far left. There were 2 additional spontaneous signals monitored during the series shown in Figure 5. One originated at the bottom center and the second originated at the upper center. Thus, the spontaneous epileptiform events

SIGNAL SPREAD
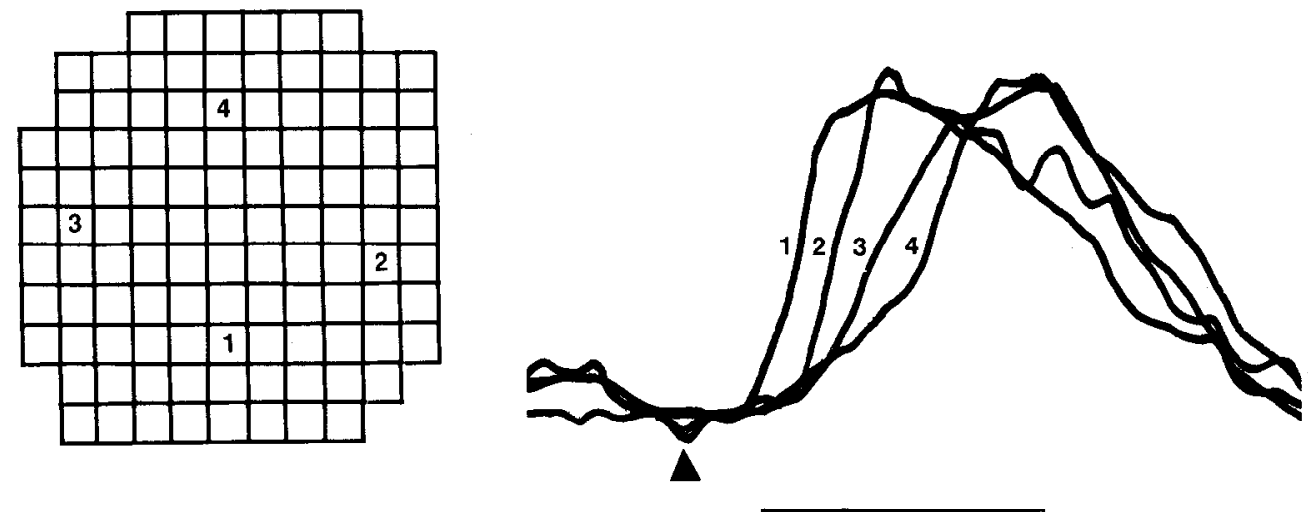

50 msec
Figure 4. Time courses of the optical signals from 4 areas of Figure 2 (bottom), after bicuculline. The signal appears first in the area of cortex $(l)$ where the control response was found (Fig. 2, top). The signal appears in the 3 other regions $(2,3$, and 4$)$ with delays between 7 and $22 \mathrm{msec}$. The time of the beginning of the whisker movement is indicated by the arrow below the traces. Each trace is the average of the signals from the 9 detectors that include and surround the numbered detector. The traces were scaled to be equal in size and filtered with 10 passes of the 1-2-1 digital smoothing routine. 
Figure 5. Comparison of 8 evoked trials recorded after the addition of bicuculline. The outputs of 104 detectors are shown for the third trial; for all other trials, the outputs of only the fourth row of detectors is shown (indicated by the large arrowheads in trial 3). In the first trial after bicucullinc, the signals were fast and localized to the right-hand portion of the array. The responses from the trials 2-8 were similar in size, spatial distribution, and time course of spread across the cortex. The first trial occurred $2 \mathrm{~min}$ after the addition of bicuculline, the second trial 6 min after the addition, and the eighth trial was $41 \mathrm{~min}$ after the addition. Not every whisker stimulation led to a large evoked signal; in about half of the attempted measurements, there were no signals on either the ball electrode or the optical rccording. The small arrowheads in the third trial indicate the beginning of the whisker movement. All the results are displayed at the same gain. For this figure, the signals were divided by the resting light levels, and thus the $\Delta F / F$ value (at right) holds for all detectors. The traces were smoothed with 5 passes of a 1-2-1 digital smoothing routine.

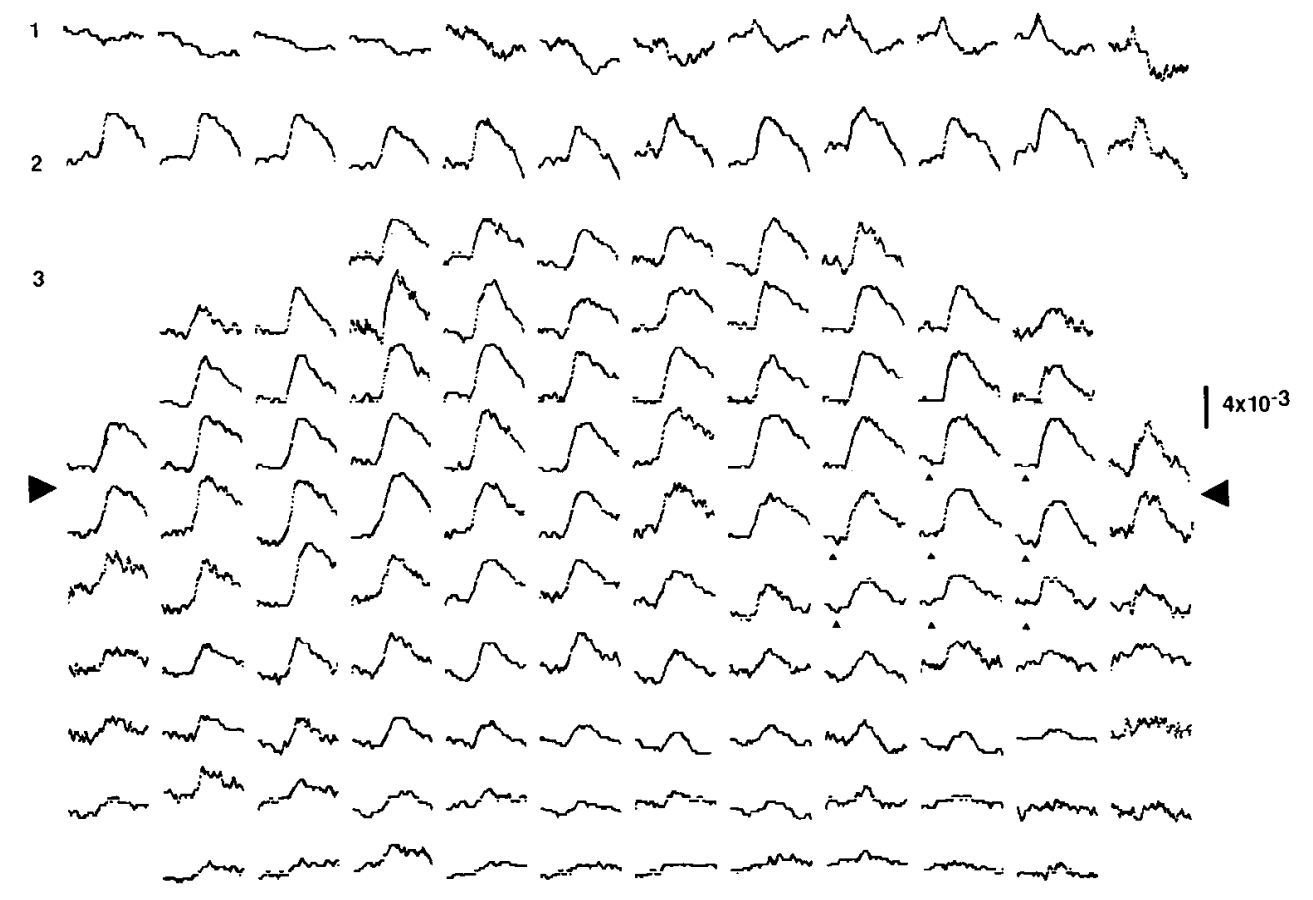

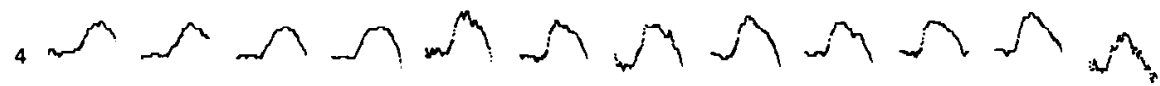

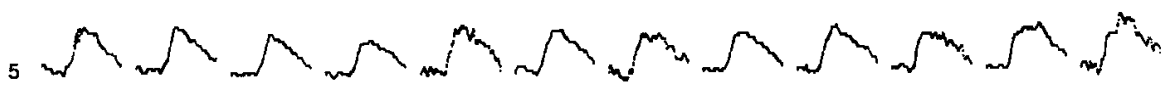

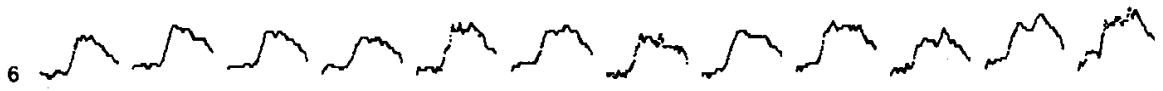

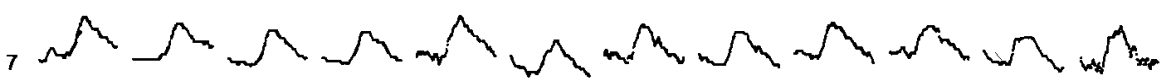

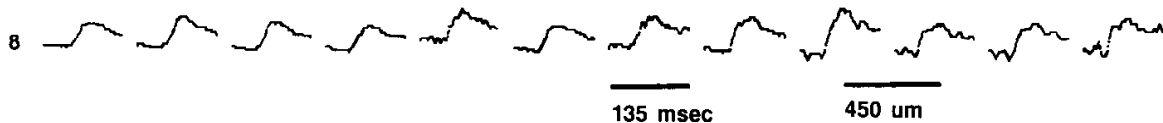

appeared to be random in their origin and variable in the extent of cortex over which they existed. They did, however, have a size, time course, and propagation velocity that was similar to the evoked epileptiform signals.

Most often the occurrence of large optical signals was correlated with large ball electrode signals. There were, however, exceptions, and instances from 2 preparations are illustrated in Figure 8. Results from experiment 16 are shown on the top and those from experiment 14 at the bottom. In each set of 3 traces, the top one is the ball electrode recording and the bottom two are representative optical recordings from separated locations on the cortex. In A and B of experiment 16 are shown 2 sequential evoked responses. While the ball electrode signals in $A$ and $B$ seem identical, in B the optical signals are much reduced in one area and absent in the second. A third recording, in C, is from a spontaneous event recorded later after reperfusion of the cortex with bicuculline. Since there is a large optical signal at this time, the absence of the optical signal in B cannot be due to bleaching of the dye or to irreversible damage to the cortex. The bottom section illustrates a similar lack of correspondence between optical and electrical recordings. In both cases the whisker movement generates an almost identical ball electrode response, but there is no detectable optical signal in $\mathrm{B}$, while relatively large signals were detected at the same location in a subsequent trial (C). Again, there was a second addition of bicuculline between $B$ and $C$. However, we are not sure that it is the addition of bicuculline per se that led to the reappearance of the signal. In some experiments, we seemed to be able to prevent the disappearance of the signal by taking more care to keep the dura moistened with saline. 


\section{Discussion}

Because both the dye concentration and the light intensity are greatest in the upper layers of cortex, the optical signals must mainly originate from cortical layers I, II, and, perhaps, III. If rat cortex had the same opacity as salamander olfactory bulb (Orbach and Cohen, 1983), then the intensity reaching $1 \mathrm{~mm}$ into cortex would be only $10 \%$ of the intensity at the surface, and similarly only $10 \%$ of the emitted light from $1 \mathrm{~mm}$ deep would reach the surface. Thus, signals from $1 \mathrm{~mm}$ deep would be attenuated by a factor of 100 . From measurements of light scattering (Orbach and Cohen, 1983), we guess that the spatial resolution of the present measurements would be in the range of a few hundred microns. Since each photodiode received light from a $450 \times 450 \mu \mathrm{m}$ area of cortex, the spatial resolution was probably limited by the number of pixels in the diode array rather than the optical properties of cortex. Even so, the spatial resolution is still greater than that obtained with cortical surface electrodes and considerably better than that obtained with scalp recordings (Cooper et al., 1965; Goldensohn and Salazar, 1986). The spatial resolution of these optical measurements could be increased by increasing the pixel number or the objective magnification.

The addition of bicuculline affected several aspects of the cortical response to whisker stimulation. The response increased in size and in duration, had a longer latency, and spread over the entire area of cortex that was treated with the drug. While the increase in latency may seem surprising, Traub and collaborators (Traub et al., 1987; Miles et al., 1988) have suggested that a signal with a long latency could occur if the event results from excitatory connections between principal cells and these connections were sparse. In this situation, the activation of a large fraction of the principal neurons would require several cycles of presynaptic spikes, excitatory postsynaptic potentials, and postsynaptic spikes.

The evoked signal found after the addition of bicuculline spread with an apparent velocity that averaged $0.6 \mathrm{~m} / \mathrm{sec}$. This velocity is within the range reported for propagation along peripheral nonmyelinated $\mathrm{C}$ fibers, $1.0 \mathrm{~m} / \mathrm{sec}$ (Douglas and Ritchie, 1961); stratum radiatum fibcrs in hippocampus, $0.5 \mathrm{~m} / \mathrm{sec}$ (Andersen et al., 1978; Miles et al., 1988); and parallel fibers in cerebellum, $0.3 \mathrm{~m} / \mathrm{sec}$ (Eccles et al., 1966). However, $0.6 \mathrm{~m} / \mathrm{sec}$ was somewhat faster than previously reported for propagation of epileptiform events in cat cortex, $0.25 \mathrm{~m} / \mathrm{sec}$ (Goldensohn and Salazar, 1986); hippocampal slices, $0.14 \mathrm{~m} / \mathrm{sec}$ (Miles et al., 1988); and neocortical slices, $0.08 \mathrm{~m} / \mathrm{sec}$ (Chervin et al., 1988). One difference between our experimental procedure and that of Goldensohn and Salazar (1986) is that they applied the epileptic agent at a small spot on the cortex and thus its concentration would have decreased with increasing distance from the point of application. If the optical signal does result from propagation along the surface of the cortex, then its velocity might suggest that propagation may be achieved by simple spread along nonmyelinated axons. It is, however, possible that the actual mechanism is not so simple (Knowles et al., 1987; Traub et al., 1987; Miles et al., 1988).

In 4 preparations a transition signal between the control response and the bicuculline treated response was seen. The transition signal (Fig. 5, trial 1; Fig. 6, first trial) was larger than the control signal, while its latency, time course, and cortical extent were similar. In subsequent trials, the evoked signal was even larger, and was also slower, delayed, and occurred over an ex-
EVOKED SIGNALS

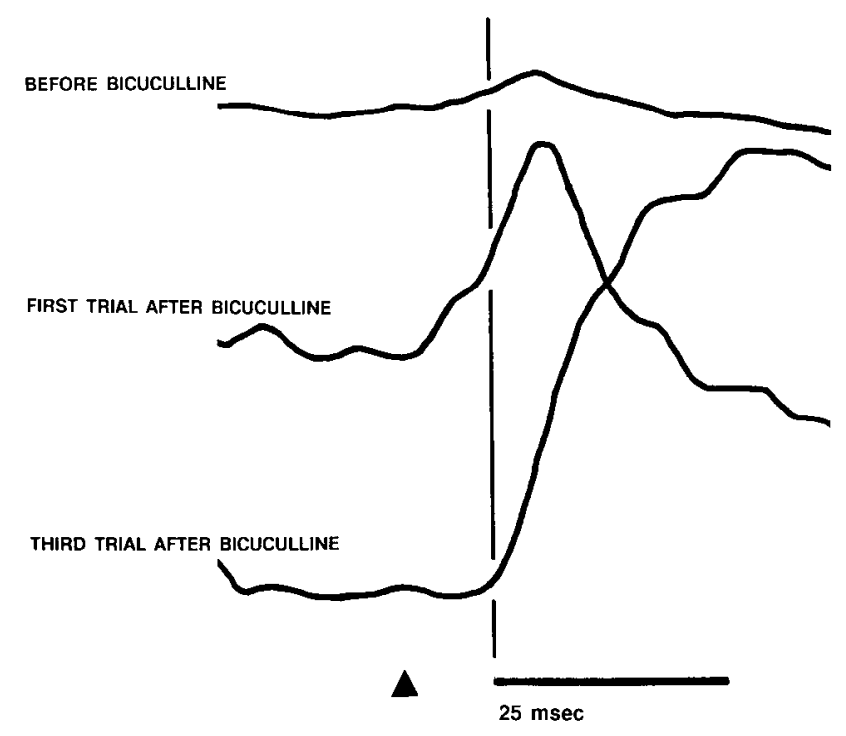

Figure 6. Comparison of the size and time course of 3 evoked signals. The control signal before the addition of bicuculline is relatively small. In the first recording after bicuculline (transition signal), the signal is larger but its time course appeared to be identical to the control signal (when both signals were scaled to the same size). In the third trial after bicuculline, the signal is even larger, but it also has a longer latency and duration. The vertical axis of 3 recordings are identical in fractional intensity change $(\Delta F / T)$. The data is from the same experiment as that illustrated in Figure 5. Each trace represents the average of the outputs of 9 detectors (the 8 indicated by the small arrows in Figure 5, trial 3, plus the detector which completes the square). The control recording was the average of 16 trials. The arrowindicates the time of the beginning of the whisker movement. The traces were smoothed with 4 passes of a 1-2-1 digital smoothing routine.

tended area of cortex. One possible interpretation of these results is that bicuculline first causes the control response to increase in size. This transition signal then converts to a signal that is slower, has a longer latency, and is more widespread. A second, alternate hypothesis is that the control signal is always present and bicuculline induces an additional signal that is large, slow, and widespread. A comparison of the control signal and the signal from the third trial after bicuculline (Fig. 6) indicates that we cannot rule out the possibility that the control signal is always present since the control signal is no bigger than the noise in the third trial. (Even averaging 6 trials after bicuculline did not improve the signal-to-noise ratio enough to rule out the continued existence of the control signal). However, in Figure 6, the transition signal does apparently disappear when the full effect of the bicuculline is seen, a result that is simply interpreted in terms of the first hypothesis. The disappearance of the transition signal makes it difficult to associate the control and transition optical signals with the control and enhanced physiological response (EPR) seen in field potential recordings (Towe et al., 1981; Ebersole and Chatt, 1986). In future experiments we plan to test other bicuculline concentrations which might lead to either more persistent or additional transition states.

After the transition, the evoked response in bicuculline appears to be relatively constant. It originates in the same location, has the same latency, and propagates over the cortex in a repeatable fashion. Furthermore these responses are approxi- 


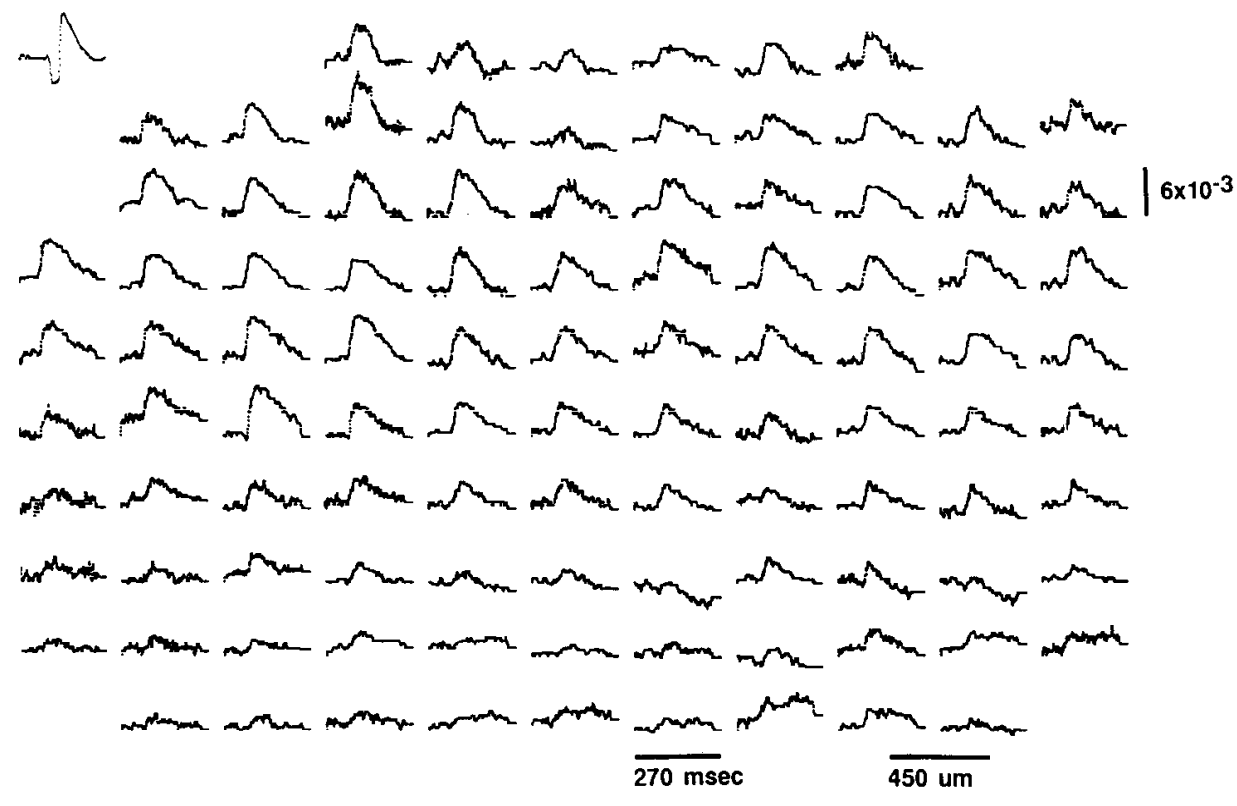

Figure 7. Two measurements made during spontaneous interictal epileptic events. In the upper panel, the epileptiform signals occur on many detectors; in the lower panel, the signal is largest in the upper right portion of the cortical àrea but cannot be detected on the left. The ball electrode recordings are shown in the upper-left corner. The 2 measurements are from the same preparation whose results are illustrated in Figures 5 and 6 and were taken during the series of evoked responses illustrated in Figure 5. For this figure, the signals were divided by the resting light levels, and thus the $\Delta F / F$ value indicated at right holds for all detectors. The traces were smoothed with 5 passes of a 1-2-1 digital smoothing routine. The frequency of spontaneous events, as detected by the ball electrode, appeared to be reduced by turning off the respirator, and thus several trials often had to be recorded to obtain a trial with a spontaneous event.

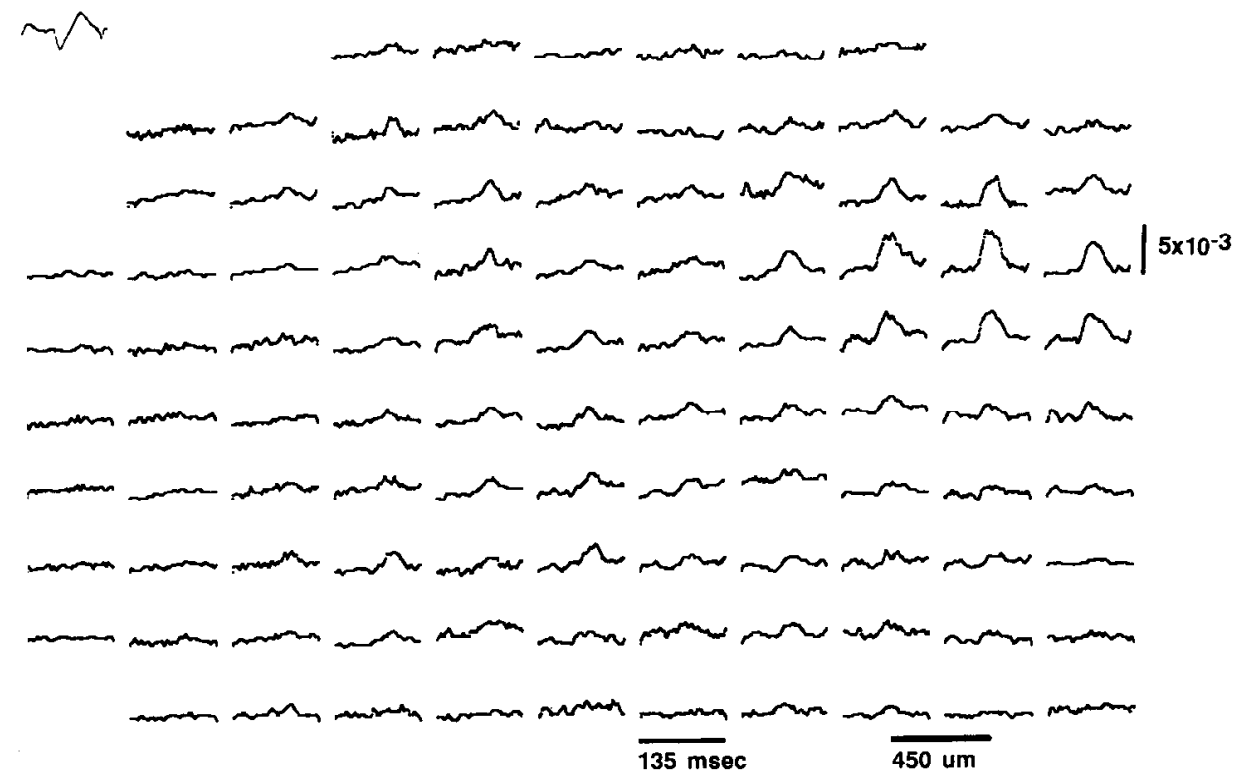

mately the same in size and time course from trial to trial (Figure 5). The consistency of the responses suggests that the effective concentration of bicuculline falls in the high range $(>10 \mu \mathrm{M})$ rather than the low range $(<1 \mu \mathrm{M})$ (Chagnac-Amitai and Connors, 1989). In contrast, the spontaneous signals seem much more variable. While they are similar to the evoked signals in size and time course, they are not fixed in their site of origin and the area of cortex over which they propagate.

In most of our measurements, there was a good correlation betwecn the ball electrode (field potential) signals and the optical recordings, but, as indicated in Figure 8, there were several instances where there were large ball electrode responses but small or undetectable optical signals. A. Arieli, R. D. Frostig, E. E. Lieke, and A. Grinvald (personal communication) found similar discrepancies between the ball electrode recordings and activity as determined by optical measurements. While we think that the optical signals are likely to originate from changes in membrane potential of the top few hundred microns of cortex, the origins of the extracellular currents recorded with ball electrodes are not easily determined (Rall and Shepherd, 1968; Creutzfeldt and Kuhnt, 1973; Llinás and Nicholson, 1974; Mitzdorf, 1985). The relationship of field potential recordings to the results obtained from intracellular electrode (or optical) measurements of membrane potential is not straightforward. For example, Goldensohn and Salazar (1986) report large and longlasting inhibitory potential changes both in the focus and in the surrounding regions of cortex, but there was no ball electrode signal at the time of these inhibitory events. The rather striking 
COMPARISON OF BALL ELECTRODE AND OPTICAL RECORDINGS

EXPERIMENT 16

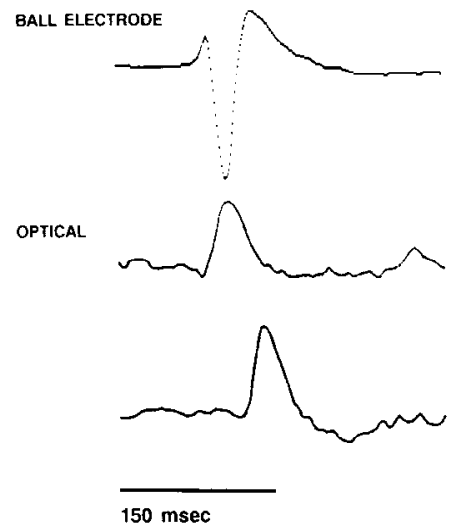

EXPERIMENT 14

$150 \mathrm{msec}$
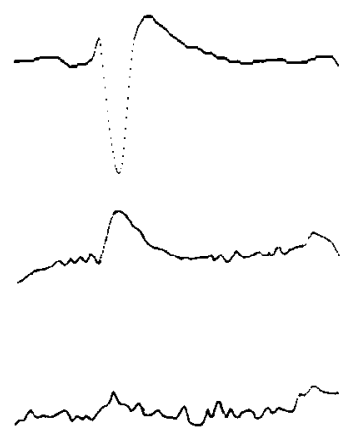
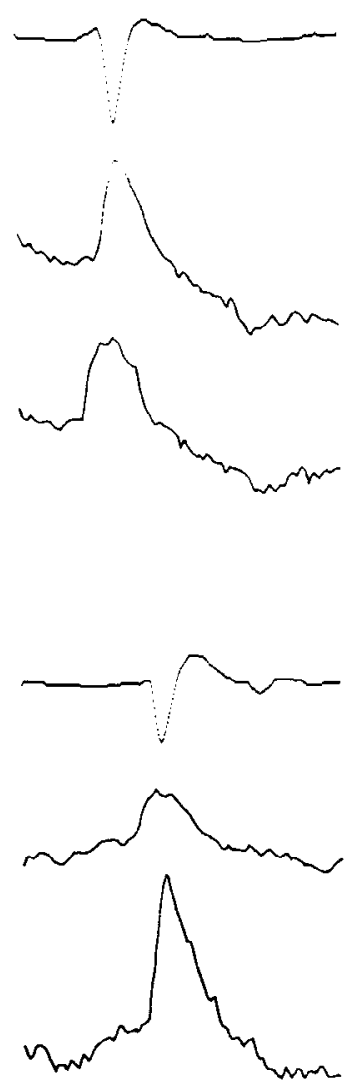

Figure 8. Comparison of ball electrode and optical recordings illustrating 2 instances where the correlation was poor. In each panel, the top trace is the ball electrode recording and the pair of optical recordings are the average of 9 detectors from 2 regions of the array. In experiment 16 , the trials shown in $A$ and $B$ have identical ball electrode responses, but the optical response in $B$ is substantially reduced. In experiment 14 , no optical response can be detected in $B$, but there is a large signal in $C$ even though the ball electrode signals are identical. The results from experiment 16 are also an example of different propagation directions for evoked $(A)$ and spontaneous $(C)$ responses. The $t o p$ optical recordings come from near the top center and the bottom optical recording came from the bottom center. The traces were smoothed with 4 passes of a 1-2-1 digital smoothing routine.

discrepancies between optical and electrode measurements i1lustrated in Figure 8 suggest caution in interpreting the optical signals in terms of signals recorded with extracellular electrodes.

The fractional changes in fluorescence $(\Delta F / F)$ from the potential-sensitive styryl dye after bicuculline were about $5 \times 10^{-3}$. These fractional changes are 1 or 2 orders of magnitude smaller than the fractional changes in NADH fluorescence that accompany interictal epileptiform discharges (Lewis et al., 1974; Schuette et al., 1974) and ictal seizures (Jobsis et al., 1971; O'Connor et al., 1972; Lewis et al., 1974; Evans and Smith, 1987). Our signals (with a styryl dye) were also 2 orders of magnitude smaller than the signals found by Evans and Smith (1987) with an oxonol dye. Part of this difference presumably results from the fact that our measurements were made during interictal events, while those of Evans and Smith were made during ictal events, and the neuronal activity during interictal events may be less intense. Furthermore, it is possible that oxonol measurements made on a slower time scale include some slow cortical signals such as those first reported by Blasdel and Salama (Blasdel and Salama, 1986; Grinvald et al., 1986). Thus, it would be of interest to test both dyes under identical measuring conditions.

The signals presented in this paper were measured with a time resolution of less than $5 \mathrm{msec}$; more than 2 orders of magnitude faster than the measurements of NADH and oxonol fluorescence (Jobsis et al., 1971; O'Connor et al., 1972; Lewis et al., 1974; Schuette et al., 1974; Evans and Smith, 1987). Since the inter-

ictal responses propagated at about $0.5 \mathrm{~mm} / \mathrm{msec}$, it is clear that millisecond time resolution is required to measure their spatial origin and propagation rates. The NADH fluorescence response to activity in the electric organ of Electrophorus has a latency of several seconds (Aubert et al., 1964); thus metabolic signals may be too slow to be useful for determining spatial origins and rates of propagation.

Because we added the bicuculline to the entire area of cortex that was exposed by the craniotomy, we could not make measurements of cortical activity at the boundary between control and bicuculline-treated cortex. In future experiments, this could be done using either local superfusion or microinjection of the epileptogenic agent (Chatt and Ebersole, 1984). Goldensohn and Salazar (1986) have presented evidence suggesting that in the region surrounding the area of bicuculline addition only a longlasting inhibition should be detected. The low-frequency filtering in the optical apparatus will have to be reduced to allow detection of signals that are this slow.

Our results suggest the possibility that optical measurements of cortical activity might be useful clinically in localizing the foci of epileptic seizures that have cortical origins or in determining the location of somatosensory or motor areas of cortex. Measurements from substantially larger areas of cortex would be needed for these applications. To make measurements from a larger area while keeping the spatial resolution constant will require an array with more pixels. Optical measurements would also be facilitated if larger signal-to-noise ratios were available. 
Larger signals could come from dyes with larger signals, or better dye delivery to neuronal membranes, or from more sophisticated noise-reduction techniques.

\section{References}

Andersen, P., H. Silfvenius, S. H. Sundberg, O. Sveen, and H. Wigstrom (1978) Functional characteristics of unmyelinated fibers in the hippocampyl cortex. Brain Res. 144: 11-18.

Aubert, X., B. Chance, and R. D. Keynes (1964) Optical studies of the biochemical events in the electric organ of Electrophorus. Proc. R. Soc. London [Biol.] 160: 211-245.

Bashford, C. L. (1981) The measurement of membrane potential using optical indicators. Biosci. Rep. I: 183-196.

Blasdel, G. G., and G. Salama (1986) Voltage-sensitive dyes reveal a modular organization in monkey striate cortex. Nature 321:579-585.

Chagnac-Amitai, Y., and B. W. Connors (1989) Horizontal spread of synchronized activity in neocortex, and its control by GABA-mediated inhibition. J. Neurophysiol. (in press).

Chatt, A. B., and J. S. Ebersole (1984) Identification of penicillin diffusion at the onset of epileptogenesis in the cat striate cortex following differential laminar microinjection. Brain Res. 290: 361-366.

Chervin, R. D., P. A. Pierce, and B. W. Connors (1988) Periodicity and directionality in the propagation of epileptiform discharges across neocortex. J. Neurophysiol. 60: 1695-1713.

Cohen, L. B., and S. Lesher (1986) Optical monitoring of membrane potential: Methods of multisite optical measurement. Soc. Gen. Physiol. Ser. 40: 71-99.

Cohen, L. B., and B. M. Salzberg (1978) Optical measurement of membrane potential. Rev. Physiol. Biochem. Pharmacol. 83: 35-88.

Cooper, R., A. L. Winter, H. J. Crow, and W. G. Walter (1965) Comparison of subcortical, cortical and scalp activity using chronically indwelling electrodes in man. Electroencephalogr. Clin. Neurophysiol. 18: 217-228.

Creutzfeldt, O. D., and U. Kuhnt (1973) Electrophysiology and topographic distribution of visual evoked potentials in animals. In Handbook of Sensory Physiology, Vol. VII/3B, R. Jung, ed., pp. 595646, Springer-Verlag, New York.

Dasheiff, R. M. (1988) Fluorescent voltage sensitive dyes: Applications for neurophysiology. J. Clin. Neurophysiol. 5: 211-235.

Douglas, W. W., and J. M. Ritchie (1961) Mammalian nonmyelinated nerve fibers. Physiol. Rev. 42: 297-334.

Ebersole, J. S., and A. B. Chatt (1986) Spread and arrest of seizures: The importance of layer 4 in laminar interactions during neocortical epileptogenesis. Adv. Neurol. 44: 515-558.

Eccles, J. C., R. Llinás, and K. Sasaki (1966) Parallel fibre stimulation and the responses induced thereby in the Purkinje cells of the cerebellum. Exp. Brain Res. 1: 17-39.

Evans, D., and J. C. Smith (1987) Seizure activity and cortical spreading depression monitored by an extrinsic potential-sensitive molecular probe. Brain Res. 409: 350-357.

Goldensohn, E. S., and A. M. Salazar (1986) Temporal and spatial distribution of intracellular potentials during generation and spread of epileptogenic discharges. Adv. Neurol. 44: 559-582.

Grinvald, A., L. B. Cohen, S. Lesher, and M. B. Boyle (1981) Simultaneous optical monitoring of activity of many neurons in invertebrate ganglia using a 124-element photodiode array. J. Neurophysiol. 45: 829-840.

Grinvald, A., E. Lieke, R. D. Frostig, C. D. Gilbert, and T. N. Wiesel (1986) Functional architecture of cortex revealed by optical imaging of intrinsic signals. Nature 324: 361-364.

Grinvald, A., R. D. Frostig, E. Lieke, and R. Hildesheim (1988) Op- tical imaging of neuronal activity. Physiol. Rev. 68: 1285-1366.

Jobsis, F. J., M. O'Connor, A. Vitale, and H. Vreman (1971) Intracellular redox changes in functioning cerebral cortex. I. Metabolic effects of epileptiform activity. J. Neurophysiol. 34: 735-749.

Knowles, W. D., R. D. Traub, and B. W. Stowbridge (1987) The initiation and spread of epileptiform bursts in the in vitro hippocampal slice. Neuroscience 21: 441-455.

Lewis, D. V., M. J. O'Connor, and W. H. Schuette (1974) Oxidative metabolism during recurrent seizures in the penicillin treated hippocampus. Electroencephalogr. Clin. Neurophysiol. 36: 347-356.

Llinás, R., and C. Nicholson (1974) Analysis of field-potentials in the central nervous system. Handbook of Electroencephalography and Clinical Neurophysiology, Vol. 2B, pp. 61-92.

London, J. A., M. Cattarelli, and L. B. Cohen (1986) The spread of epileptiform discharge in the somatosensory cortex of the marine rat measured with voltage sensitive dyes. Biol. Bult. (Abstr.) 171: 496.

London, J. A., J.-Y. Wu, M. Rioult, M. Cattarelli, and L. B. Cohen (1987) The spread of epileptiform discharges in rat somatosensory cortex measured with a voltage sensitive dye. Soc. Neurosci. Abstr. 13: 365

Miles, R., R. D. Traub, and R. K. S. Wong (1988) Spread of synchronous firing in longitudinal slices from the CA3 region of the hippocampus. J. Neurophysiol. 60: 1481-1496.

Mitzdorf, U. (1985) Current source-density method and application in cat cerebral cortex: Investigation of evoked potentials and EEG phenomena. Physiol. Rev. 65: 37-100.

O'Connor, M. J., C. J. Herman, M. Rosenthal, and F. F. Jöbsis (1972) Intracellular redox changes preceding onset of epileptiform activity in intact cat hippocampus. J. Neurophysiol. 35: 471-483.

Orbach, H. S. (1988) Monitoring electrical activity in rat cerebral cortex. In Spectroscopic Membrane Probes, Vol. 3, L. M. Loew, ed., pp. 115-135, CRC, Boca Raton, FL.

Orbach, H. S., and L. B. Cohen (1983) Optical monitoring of activity from many areas of the in vitro and in vivo salamander olfactory bulb: A new method for studying functional organization in the vertebrate central nervous system. J. Neurosci. 3: 2251-2262.

Orbach, H. S., L. B. Cohen, and A. Grinvald (1985) Optical mapping of electrical activity in rat somatosensory and visual cortex. J. Neurosci. 5: 1886-1895.

Rall, W., and G. M. Shepherd (1968) Theoretical reconstruction of field potentials and dendrodendritic synaptic interactions in olfactory bulb. J. Neurophysiol. 31: 884-915.

Rosenthal, M., and F. F. Jobsis (1971) Intracellular redox changes in functioning cerebral cortex. II. Effects of direct cortical stimulation. J. Neurophysiol. 34: 750-762.

Salzberg, B. M. (1983) Optical recording of electrical activity in neurons using molecular probes. In Current Methods in Cellular Neurobiology, J. L. Barker and J. F. McKelvy, eds., pp. 139-187, Wiley, New York.

Schuette, W. H., W. C. Whitehouse, D. V. Lewis, M. O'Connor, and J. M. Van Buren (1974) A television fluorimeter for monitoring oxidative metabolism in intact tissue. Med. Instrum. 8: 331-333.

Towe, A. L., M. D. Mann, and G. W. Harding (1981) On the currents that flow during the strychnine spike. Electroencephalogr. Clin. Neurophysiol. 51: 306-327.

Traub, R. D., W. D. Knowles, R. Miles, and R. K. S. Wong (1987) Models of the cellular mechanism underlying propagation of epileptiform activity in the CA2-CA3 region of the hippocampal slice. Neuroscience 21: 457-470.

Zemon, V., E. Kaplan, and F. Katliff (1980) Bicuculline enhances a negative component and diminishes a positive component of the visual evoked cortical potential in the cat. Proc. Natl. Acad. Sci. USA 77: 7476-7478. 